\title{
Potential roles for kinesins at the cortical division site
}

\section{Elisabeth Lipka and Sabine Müller*}

Cell and Developmental Genetics, Center for Plant Molecular Biology, University of Tuebingen, Tuebingen, Germany

Edited by:

Luis Vidali, Worcester Polytechnic

Institute, USA

\section{Reviewed by:}

Pankaj Dhonukshe, Utrecht

University, Netherlands

Giampiero Cai, Università degli Studi

di Siena, Italy

\section{*Correspondence:}

Sabine Müller, Cell and Developmental Genetics, Center for Plant Molecular Biology, University of Tuebingen, Auf der Morgenstelle 3, D-72076

Tuebingen, Germany. e-mail:

sabine.mueller@zmbp.uni-tuebingen.de
Spatial control of cytokinesis is critical for cell and plant morphology. The plane of cell division is established at G2/M transition and is initially demarcated at the cortex of the cell by the cytoskeletal preprophase band (PPB) and subsequently throughout mitosis by the cortical division zone (CDZ). Few kinesins, belonging to different classes of the superfamily, either display a distinct spatio-temporal localization at the PPB and CDZ, or genetic evidence proposes a specific function there. Protein phosphorylation and degradation are likely directing the cell cycle-dependent localization and activity of some of these kinesins, as indicated by mutation of respective conserved motifs. Furthermore, kinesins are required for continuous recruitment of CDZ identity markers to the CDZ. This review summarizes the limited current knowledge of kinesins potentially involved in the steps required for correctly oriented division planes, considering localization patterns and genetic evidence, and discussing kinesin function in context with interaction partners and cell cycle regulation.

Keywords: kinesin, cell division, cortical division site, preprophase band, phragmoplast

\section{INTRODUCTION}

The cytoskeleton is a major facilitator of cell division and cell expansion in all organisms. In cellulose-enfolded plant cells, specific cytoskeletal arrays are responsible for the selection of the division plane in pre-mitotic cells and the formation of the cell plate to partition cytoplasmic contents of daughter cells during cytokinesis. The preprophase band ( $\mathrm{PPB})$, composed of microtubules (MTs), F-actin, and endoplasmic reticulum delineates the plane of cell division at the cell cortex. The transition from the interphase cortical MT array to the mitotic PPB involves local changes in MT dynamic behavior, regulated by the activity of MTassociated proteins (MAPs; Dhonukshe and Gadella, 2003; Vos et al., 2004). Minute detail is known about the regulation of PPB assembly; however, genes encoding MT nucleation factors TONNEAU (TON) $1 \mathrm{~A}$ and TON1B and a protein phosphatase PP2A subunit TON2/DCD1/ADD1 are strictly required for PPB formation in Arabidopsis, Physcomitrella and maize, respectively, since knockout mutants lack PPBs and exhibit mis-positioned division planes (Camilleri et al., 2002; Azimzadeh et al., 2008; Wright et al., 2009; Spinner et al., 2010). The PPB's spatial information is preserved throughout mitosis by proteins, distinctly recruited to the cortical division zone (CDZ), formerly occupied by the PPB, and by proteins selectively depleted from the CDZ. Thus, the CDZ is tagged by positive and negative identity markers. Progressive confinement of the $\mathrm{CDZ}$ during cytokinesis specifies the precise site of cell plate fusion, the cortical division site (CDS).

Among the 61 predicted kinesins in Arabidopsis, only about one-third were up-regulated during mitosis (Menges et al., 2003; Lee and Liu, 2004; Vanstraelen et al., 2006a) and even fewer were implicated in division plane selection and maintenance (Zhu and Dixit, 2011).

\section{DIVISION PLANE SELECTION AND PPB FORMATION}

The position of the nucleus is informative for division plane orientation (Muller, 2011; Rasmussen et al., 2011a). Displacement of the prophase nucleus in protonemata leads to the formation of a new PPB, encircling the dislodged nucleus (Murata and Wada, 1991). Prior to proliferative, symmetric divisions in pre-mitotic cells, the nucleus is centered presumably by MT length-dependent forces (Goodbody et al., 1991; Besson and Dumais, 2011).

Recently, members of the $\mathrm{KCH}$ subgroup of kinesin-14 class were implicated in nuclear migration. A number of $\mathrm{KCH}$ proteins tested so far displayed actin binding activity conferred by the conserved Calponin homology $(\mathrm{CH})$ domain (Figure 2) potentially linking the MT and the actin cytoskeleton (Frey et al., 2009; Buschmann et al., 2011; Klotz and Nick, 2012). A study revealed the existence of motile, MT-associated NtKCH populations at the cell cortex, and non-motile peri-nuclear populations associating with actin in interphase of tobacco BY-2 cells (Klotz and Nick, 2012). Consistent with overexpression of its Arabidopsis homolog AtKinG in BY-2 cells and with other kinesins in that class, $\mathrm{NtKCH}$ motility was MT minus end directed (Lee and Liu, 2004; Buschmann et al., 2011). Furthermore, motile NtKCH associated with a subset of MTs, bridging the nucleus with the cell cortex. Thus, it was suggested that $\mathrm{KCH}$ might act in the positioning of the nucleus involving a combination of MT dynamics and actin anchored KCH sliding toward MT minus ends (Klotz and Nick, 2012). Indeed, pre-mitotic nuclear migration was significantly delayed in tobacco BY- 2 cells overexpressing GFP-KCH1 from rice ( $\mathrm{OsKCH} 1$; Frey et al., 2010).

Kinesin-14 class members ATK1 and KCBP and kinesin-5 AtKRP125c co-localized with the PPB, but also with interphase and mitotic MT arrays supporting a more general role in MT bundling (Bowser and Reddy, 1997; Marcus et al., 2003; Bannigan et al., 2007). Although atk1 mutants display wider PPBs indicating a role in PPB formation, an impact on the CDZ or cell wall positioning was not reported (Marcus et al., 2003).

The function of the negative $\mathrm{CDZ}$ marker, kinesin KCA1 remains enigmatic (Vanstraelen etal., 2006b). KCA1 accumulated at the plasma membrane at high levels during mitosis, but 
remained absent from the CDZ, presenting a KCA1 depleted site and resembling aspects of F-actin distribution (Figure 1). As indicated by MT-depolymerization experiments, formation of the KCA1 depleted site depended on prior PPB assembly and absence of the KCA1 depleted site lead to mis-positioning of cell plates. Similarly, drug induced depolymerization of F-actin before formation of the actin depleted zone/microfilament twin peaks (ADZ/MFTP) disturbed proper cell plate positioning in BY-2 cells (Hoshino et al., 2003; Sano et al., 2005). In contrast, KCA1 localization did not alter upon actin depolymerization (Vanstraelen et al., 2006b). Interestingly, kcalkca2 double mutants were defective in light-induced chloroplast movement (Suetsugu et al., 2010), a process known to be actin dependent. It is likely that the $\mathrm{CDZ}$ requires an environment of reduced motility to recruit and maintain a certain suite of proteins (Panteris, 2008). Experiments pertaining to the temporal relations between KCA1 and other CDZ markers should help elucidate the significance of differential KCA1 localization.

KCA1 and its homolog KCA2 were initially identified as CDKA;1 interaction partners (Vanstraelen et al., 2004). The KCAs shared a conserved domain structure with N-terminal motor domain. However, the motor domain was most similar to that of C-terminal kinesins and preceded by a neck-linker and therefore, KCAs were placed within the kinesin-14 subfamily (Vanstraelen et al., 2004). The subsequent stalk domain-mediated homo- and hetero-dimerization in vitro (Vanstraelen et al., 2004) and the C-terminal tails of KCA1 and KCA2 featured three and two CDKA;1 phosphorylation sites, respectively. Site directed mutagenesis indeed reduced binding to CDKA;1 in vitro and intramolecular folding of the tail onto the stalk was obstructed. Thus, KCA activity might be regulated depending on their phosphorylation status, which might be addressed by expression of phospho-mimic mutants in plants.

Progression through the cell cycle depends on the timely degradation of cell cycle regulatory proteins, to ensure synchronization of chromatin condensation and mitotic cytoskeletal array formation. Recently, the kinesin AtKINUa/ARK3 was proposed to act as a synchronizer (Sakai et al., 2008; Malcos and Cyr, 2011). AtKINUa is a member of a small ungrouped class of kinesins, present in plants and protists. Their domain structure is unique, comprising

A
Interphase
Preprophase
G1/G2
prophase
Prometaphase
NEB
Mitosis
Termination of

midplane

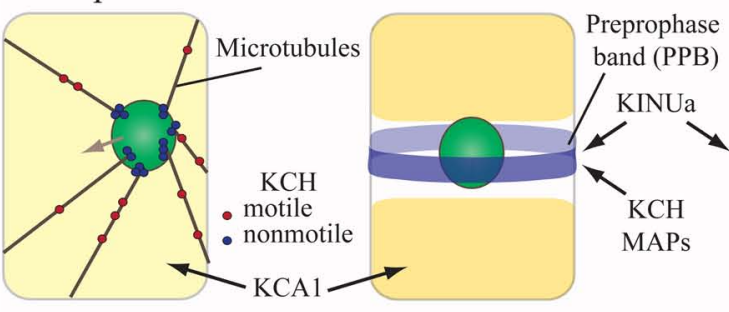

Nuclear

migration

Selection
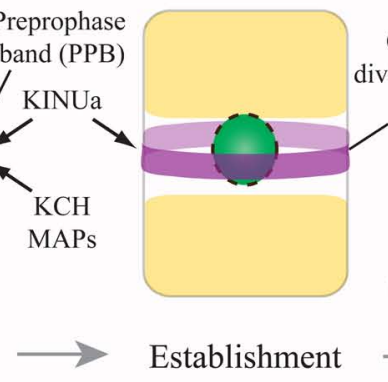

Cortical
vision zone
$(C D Z)$ (CDZ)
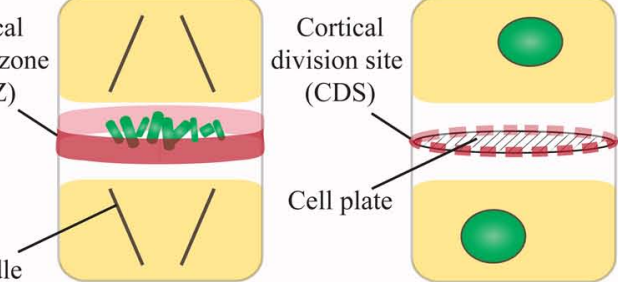

Spindle

Establishment

Maintenance
recognition

Cell plate

insertion
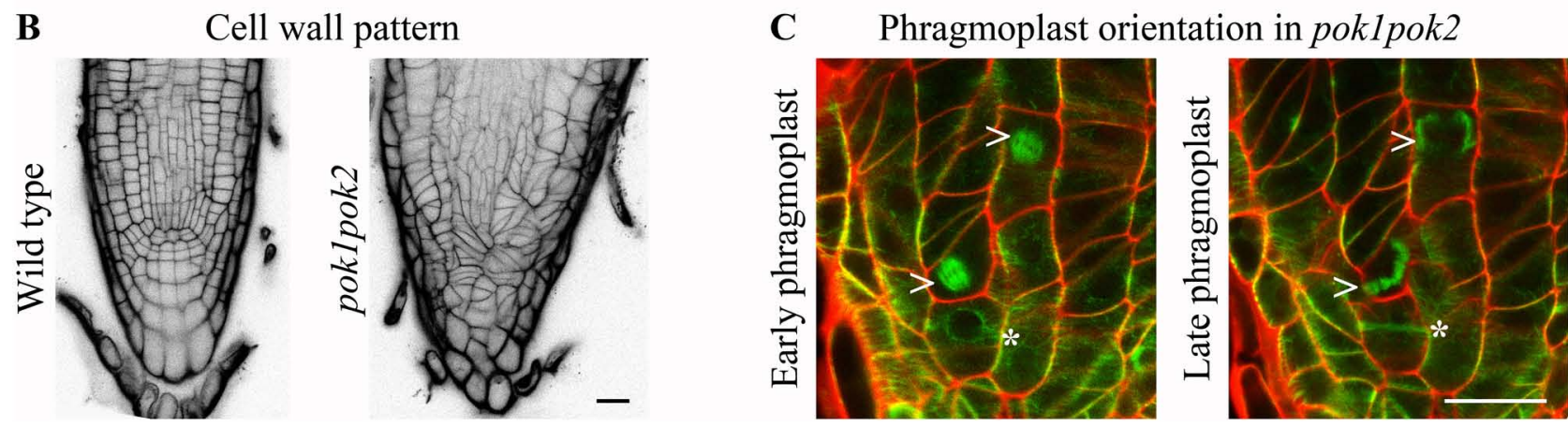

FIGURE 1 | Kinesins at the cortical division zone (CDZ). (A) In pre-mitotic cells, nuclear migration to the cell center is accomplished by microtubule (MT) force-dependent sensing of cell geometry potentially mediated by $\mathrm{KCH}$ kinesins. The preprophase band (PPB) circumscribes the future division plane at the cell cortex. Several MT-associated proteins (MAPs) and kinesin AtKINUa and the Arabidopsis KCH (AtKinG) are recruited to the $\mathrm{PPB} / \mathrm{CDZ}$, while the plasma membrane resident

kinesin KCA1 becomes depleted from this site. Nucleus/chromosomes are indicated in green. (B and $\mathbf{C}$ ) Confocal micrographs of seedling root tips. (B) Wild type and pok1pok2 cell wall patterns, visualized by Propidium lodide (PI) staining. (C) Change of phragmoplast (arrow head) orientation from a transverse to orthogonal plane in pok1pok2. Asterisk indicates PPB. Green label: GFP-MT marker, red label: cell wall by PI staining. Scale bar $20 \mu \mathrm{m}$. 
a non-conserved $\mathrm{N}$-terminal motor domain and a variable number of armadillo repeats at their C-terminus, however, lacking a characteristic neck-linker (Malcos and Cyr, 2011). Furthermore, these kinesins contain a conserved destruction box (D-BOX) motif, serving as a potential target for proteasome-mediated degradation. AtKINUa associated with cortical MTs in interphase, but became highly enriched at the PPB in prophase and eventually disappeared upon nuclear envelope breakdown (NEB) in metaphase. Intriguingly, not only AtKINUa degradation at the NEB depended on the D-BOX motif, but also the protein's association with PPB MTs. The mutation of a conserved residue within the D-BOX motif resulted in diffuse accumulation of AtKINUa-GFP at the PPB, as well as at the spindle and phragmoplast, indicating that timely degradation of the fusion protein was obstructed (Malcos and Cyr, 2011). Immediately succeeding the D-BOX is a putative CDKA phosphorylation site, however, its significance for AtKINUa localization was not evaluated so far. AtKINUa was distinctly expressed in embryos and cells of the stomatal lineage in Arabidopsis, but, genetic evidence for specific function of AtKINUa during cell division is not available yet (Sakai et al., 2008; Malcos and Cyr, 2011).

The confined spatio-temporal localization pattern of AtKINUa and its protein domains narrow the number of potential interaction partners to several PPB-associated MAPs such as MOR1, CLASP, AIR9, or MAP65s (Buschmann et al., 2006; Kawamura etal., 2006; Ambrose et al., 2007) and above mentioned kinesins ATK1, KCBP, and AtKRP125c. TON2/DCD1, however, showed a spatio-temporal distribution very similar to AtKINUa (Wright etal., 2009) making it a probable interaction partner. Indeed, recently shown genetic interaction between TON2 and MOR1 and TON2 and TON1 further validated the involvement of these proteins in division plane placement (Kirik et al., 2012). Most likely, TON2-dependent dephosphorylation modulates the activity of MOR1 and TON1 in MT array organization. Thus, functional aspects of AtKINUa might be revealed by investigating localization of endogenous and AtKINUa phosphomutants in mutants defective in PPB formation and cell wall positioning.

\section{DIVISION PLANE MAINTENANCE}

PHRAGMOPLAST ORIENTING KINESIN (POK) 1 and 2 were required for the preservation and/or recognition of spatial information conveyed by the PPB. POKs belonged to the kinesin-12 class based on their N-terminal motor domain and were the largest predicted kinesins in Arabidopsis (Figure 2; Lee and Liu, 2004). Despite their significant size difference (Figure 2) and overlapping, yet distinct gene expression patterns, POK1 and POK2 exhibited functional redundancy. While single mutants were indistinguishable from wild-type, double mutants of T-DNA insertion alleles displayed dwarfed overall morphology and pronounced mis-orientation of cell walls in root meristems, deviating from the regular pattern characteristic for wild-type (Figure 1B; Muller et al., 2006).

The plant-specific MAP TANGLED and the Ran regulatory protein RanGAP1, both positive CDZ identity markers, colocalized with the PPB and the CDZ throughout mitosis. TAN as well as RanGAP1 fusion proteins were inadequately recruited to the PPB in pok1pok2 (Walker et al., 2007; Xu et al., 2008). Moreover, TAN and RanGAP1 association with the CDZ was not maintained past metaphase, revealing the dynamic nature of the CDZ. Strikingly, pok1pok2 phragmoplasts appeared to lack guidance (Figure 1C) and the cell plate fused with the parental cell wall seemingly random, wherever they encountered upon completion of cytokinesis. Since the initial recruitment of TAN and RanGAP1 to the PPB occurred independent of POKs (Xu et al., 2008; Rasmussen et al., 2011b), kinesins other than POK1 and POK2 might support this task. The C-terminus of POK1mediated interaction with both, TAN and RanGAP1 (Muller et al., 2006; Xu et al., 2008), suggesting that POK1 itself localized to the CDZ.

$\begin{aligned} & \text { Kinesin } \\ & \text { family }\end{aligned}$ Kinesin
KOK1
ungrouped KINUa




\section{CONCLUDING REMARKS}

KCAs and AtKINUa are documented instances for the impact of cell cycle-dependent phospho-regulation and protein degradation on protein activity and localization. Notably, the CDK consensus motif [S/T-P-x-K/R] was detected several times in POKs, in AtKinG $(\mathrm{KCH})$ and a phosphorylation site was predicted for AtKINUa (Malcos and Cyr, 2011). Furthermore, consensus D-BOX motifs $\left[\mathrm{R}-\mathrm{x}_{2}-\mathrm{L}-\mathrm{x}_{4}-\mathrm{N} / \mathrm{Q}\right]$ were present in POKs (manual annotation and Vanstraelen et al., 2006a).

So far, motility was only reported for $\mathrm{KCH}$ kinesins. Future research assignments certainly involve in vivo live-cell imaging studies as well as in vitro assembly and imaging to investigate

\section{REFERENCES}

Ambrose, J. C., Shoji, T., Kotzer, A. M., Pighin, J. A., and Wasteneys, G. O. (2007). The Arabidopsis CLASP gene encodes a microtubule-associated protein involved in cell expansion and division. Plant Cell 19, 2763-2775.

Azimzadeh, J., Nacry, P., Christodoulidou, A., Drevensek, S., Camilleri, C., Amiour, N., Parcy, F., Pastuglia, M., and Bouchez, D. (2008). Arabidopsis TONNEAU1 proteins are essential for preprophase band formation and interact with centrin. Plant Cell 20, 2146-2159.

Bannigan, A., Scheible, W. R., Lukowitz, W., Fagerstrom, C., Wadsworth, P., Somerville, C., and Baskin, T. I. (2007). A conserved role for kinesin5 in plant mitosis. J. Cell Sci. 120, 2819-2827.

Besson, S., and Dumais, J. (2011). Universal rule for the symmetric division of plant cells. Proc. Natl. Acad. Sci. U.S.A. 108, 6294-6299.

Bowser, J., and Reddy, A. S. (1997). Localization of a kinesin-like calmodulin-binding protein in dividing cells of Arabidopsis and tobacco. Plant J. 12, 1429-1437.

Buschmann, H., Chan, J., SanchezPulido, L., Andrade-Navarro, M. A., Doonan, J. H., and Lloyd, C. W. (2006). Microtubule-associated AIR9 recognizes the cortical division site at preprophase and cell-plate insertion. Curr. Biol. 16, 1938-1943.

Buschmann, H., Green, P., Sambade, A., Doonan, J. H., and Lloyd, C. W. (2011). Cytoskeletal dynamics in interphase, mitosis and cytokinesis analysed through Agrobacteriummediated transient transformation of tobacco BY-2 cells. New Phytol. 190, 258-267.

Camilleri, C., Azimzadeh, J., Pastuglia, M., Bellini, C., Grandjean, O., and Bouchez, D. (2002). The Arabidopsis TONNEAU2 gene encodes a putative novel protein phosphatase $2 \mathrm{~A}$ regulatory subunit essential for the control of the cortical cytoskeleton. Plant Cell 14, 833-845.
Dhonukshe, P., and Gadella, T. W., Jr. (2003). Alteration of microtubule dynamic instability during preprophase band formation revealed by yellow fluorescent protein-CLIP170 microtubule plus-end labeling. Plant Cell 15, 597-611.

Frey, N., Klotz, J., and Nick, P. (2009). Dynamic bridges - a calponin-domain kinesin from rice links actin filaments and microtubules in both cycling and noncycling cells. Plant Cell Physiol. 50, 1493-1506.

Frey, N., Klotz, J., and Nick, P. (2010). A kinesin with calponin-homology domain is involved in premitotic nuclear migration. J. Exp. Bot. 61, 3423-3437.

Goodbody, K. C., Venverloo, C. J., and Lloyd, C. W. (1991). Laser microsurgery demonstrates that cytoplasmic strands anchoring the nucleus across the vacuole of premitotic plant cells are under tension. Implications for division plane alignment. Development 113, 931-939.

Hoshino, H., Yoneda, A., Kumagai, F., and Hasezawa, S. (2003). Roles of actin-depleted zone and preprophase band in determining the division site of higher-plant cells, a tobacco BY-2 cell line expressing GFP-tubulin. Protoplasma 222, 157-165.

Kawamura, E., Himmelspach, R., Rashbrooke, M. C., Whittington, A. T., Gale, K. R., Collings, D. A., and Wasteneys, G. O. (2006). MICROTUBULE ORGANIZATION 1 regulates structure and function of microtubule arrays during mitosis and cytokinesis in the Arabidopsis root. Plant Physiol. 140, 102-114.

Kirik, A., Ehrhardt, D. W., and Kirik, V. (2012). TONNEAU2/FASS regulates the geometry of microtubule nucleation and cortical array organization in interphase Arabidopsis cells. Plant Cell 24, 1158-1170.

Klotz, J., and Nick, P. (2012). A novel actin-microtubule cross-linking

kinetics of kinesins taking part in CDZ selection and maintenance. However, successful analysis depends on the availability of functional fusion proteins which may be confirmed by complementation of mutants or consensus localization with specific antibodies. Furthermore, the low abundance of these kinesins poses challenges, even for sensitive imaging systems. Nevertheless, recent technical advances in imaging may contribute to tackle these objectives.

\section{ACKNOWLEDGMENT}

Work in this field of research is supported by the Deutsche Forschungsgemeinschaft (Project MU3133/1-1 to Sabine Müller).

kinesin, NtKCH, functions in cell expansion and division. New Phytol. 193, 576-589.

Lawrence, C. J., Dawe, R. K., Christie, K. R., Cleveland, D. W., Dawson, S. C., Endow, S. A., Goldstein, L. S., Goodson, H. V., Hirokawa, N., Howard, J., Malmberg, R. L., Mcintosh, J. R., Miki, H., Mitchison, T. J., Okada, Y., Reddy, A. S., Saxton, W. M., Schliwa, M., Scholey, J. M., Vale, R. D., Walczak, C. E., and Wordeman, L. (2004). A standardized kinesin nomenclature. J. Cell Biol. 167, 19-22.

Lee, Y. R. J., and Liu, B. (2004). Cytoskeletal motors in Arabidop sis. Sixty-one kinesins and seventeen myosins. Plant Physiol. 136, 3877-3883.

Malcos, J. L., and Cyr, R. J. (2011) An ungrouped plant kinesin accumulates at the preprophase band in a cell cycle-dependent manner. Cytoskeleton (Hoboken) 68, 247-258.

Marcus, A. I., Li, W., Ma, H., and Cyr, R. J. (2003). A kinesin mutant with an atypical bipolar spindle undergoes normal mitosis. Mol. Biol. Cell 14, 1717-1726.

Menges, M., Hennig, L., Gruissem, W., and Murray, J. A. (2003). Genomewide gene expression in an Arabidopsis cell suspension. Plant Mol. Biol. 53 , 423-442.

Muller, S. (2011). Universal rules for division plane selection in plants. Protoplasma 249, 239-253.

Muller, S., Han, S., and Smith, L. G. (2006). Two kinesins are involved in the spatial control of cytokinesis in Arabidopsis thaliana. Curr. Biol. 16, 888-894.

Murata, T., and Wada, M. (1991). Effects of centrifugation on preprophaseband formation in Adiantum protonemata. Planta 183, 391-398.

Panteris, E. (2008). Cortical actin filaments at the division site of mitotic plant cells: a reconsideration of the 'actin-depleted zone'. New Phytol. 179, 334-341.
Rasmussen, C. G., Humphries, J. A., and Smith, L. G. (2011a). Determination of symmetric and asymmetric division planes in plant cells. Annu. Rev. Plant Biol. 62, 387-409.

Rasmussen, C. G., Sun, B., and Smith, L. G. (2011b). Tangled localization at the cortical division site of plant cells occurs by several mechanisms. J. Cell Sci. 124, 270-279.

Sakai, T., Honing, H. V. D., Nishioka, M., Uehara, Y., Takahashi, M., Fujisawa, N., Saji, K., Seki, M., Shinozaki, K., Jones, M. A., Smirnoff, N., Okada, K., and Wasteneys, G. O. (2008). Armadillo repeat-containing kinesins and a NIMA-related kinase are required for epidermal-cell morphogenesis in Arabidopsis. Plant J. 53, 157-171.

Sano, T., Higaki, T., Oda, Y., Hayashi, T., and Hasezawa, S. (2005). Appearance of actin microfilament 'twin peaks' in mitosis and their function in cell plate formation, as visualized in tobacco BY-2 cells expressing GFP-fimbrin. Plant J. 44, 595-605.

Spinner, L., Pastuglia, M., Belcram, K., Pegoraro, M., Goussot, M., Bouchez, D., and Schaefer, D. G. (2010). The function of TONNEAU1 in moss reveals ancient mechanisms of division plane specification and cell elongation in land plants. Development 137, 2733-2742.

Suetsugu, N., Yamada, N., Kagawa, T., Yonekura, H., Uyeda, T. Q. P., Kadota, A., and Wada, M. (2010). Two kinesin-like proteins mediate actin-based chloroplast movement in Arabidopsis thaliana. Proc. Natl. Acad. Sci. U.S.A. 107, 8860-8865.

Vanstraelen, M., Inzé, D., and Geelen, D. (2006a). Mitosis-specific kinesins in Arabidopsis. Trends Plant Sci. 11, 167-175.

Vanstraelen, M., Torres Acosta, J. A., De Veylder, L., Inze, D., and Geelen, D. (2004). A plant-specific subclass of C-terminal kinesins contains a conserved a-type cyclin-dependent 
kinase site implicated in folding and dimerization. Plant Physiol. 135, 1417-1429.

Vanstraelen, M., Van Damme, D., De Rycke, R., Mylle, E., Inze, D., and Geelen, D. (2006b). Cell cycle-dependent targeting of a kinesin at the plasma membrane demarcates the division site in plant cells. Curr. Biol. 16, 308-314.

Vos, J. W., Dogterom, M., and Emons, A. M. (2004). Microtubules become more dynamic but not shorter during preprophase band formation: a possible "search-and-capture" mechanism for microtubule translocation. Cell Motil. Cytoskeleton 57, 246-258.
Walker, K. L., Muller, S., Moss, D., Ehrhardt, D. W., and Smith, L. G. (2007). Arabidopsis TANGLED identifies the division plane throughout mitosis and cytokinesis. Curr. Biol. 17, 1827-1836.

Wright, A. J., Gallagher, K., and Smith, L. G. (2009). discordial and alternative discordial function redundantly at the cortical division site to promote preprophase band formation and orient division planes in maize. Plant Cell 21, 234-247.

Xu, X. M., Zhao, Q., Rodrigo-Peiris, T., Brkljacic, J., He, C. S., Muller, S., and Meier, I. (2008). RanGAP1 is a continuous marker of the
Arabidopsis cell division plane. Proc. Natl. Acad. Sci. U.S.A. 105, 1863718642.

Zhu, C., and Dixit, R. (2011). Functions of the Arabidopsis kinesin superfamily of microtubule-based motor proteins. Protoplasma doi: 10.1007/s00709-011-0343-9 [Epub ahead of print].

Conflict of Interest Statement: The authors declare that the research was conducted in the absence of any commercial or financial relationships that could be construed as a potential conflict of interest.
Received: 30 April 2012; accepted: 28 June 2012; published online: 13 July 2012.

Citation: Lipka E and Müller S (2012)

Potential roles for kinesins at the cortical division site. Front. Plant Sci. 3:158. doi: 10.3389/fpls.2012.00158

This article was submitted to Frontiers in Plant Cell Biology, a specialty of Frontiers in Plant Science.

Copyright (C) 2012 Lipka and Müller. This is an open-access article distributed under the terms of the Creative Commons Attribution License, which permits use, distribution and reproduction in other forums, provided the original authors and source are credited and subject to any copyright notices concerning any thirdparty graphics etc. 